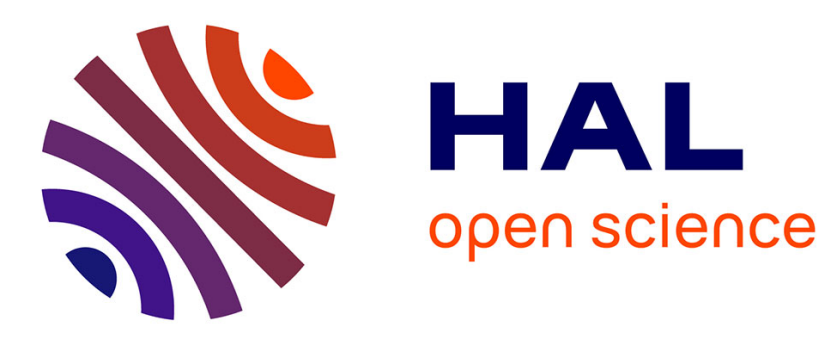

\title{
Mu-Calculus Path Checking
}

Nicolas Markey, Philippe Schnoebelen

\section{To cite this version:}

Nicolas Markey, Philippe Schnoebelen. Mu-Calculus Path Checking. Information Processing Letters, 2006, 97 (6), pp.225-230. 10.1016/j.ipl.2005.11.010 . hal-01194606

\section{HAL Id: hal-01194606 https://hal.science/hal-01194606}

Submitted on 7 Sep 2015

HAL is a multi-disciplinary open access archive for the deposit and dissemination of scientific research documents, whether they are published or not. The documents may come from teaching and research institutions in France or abroad, or from public or private research centers.
L'archive ouverte pluridisciplinaire HAL, est destinée au dépôt et à la diffusion de documents scientifiques de niveau recherche, publiés ou non, émanant des établissements d'enseignement et de recherche français ou étrangers, des laboratoires publics ou privés. 


\title{
Mu-calculus path checking
}

\author{
Nicolas Markey and Philippe Schnoebelen \\ Lab. Spécification $\&$ Vérification, CNRS \& ENS de Cachan, France
}

\begin{abstract}
We investigate the path model checking problem for the $\mu$-calculus. Surprisingly, restricting to deterministic structures does not allow for more efficient model checking algorithm, as we prove that it can encode any instance of the standard model checking problem for the $\mu$-calculus.
\end{abstract}

\section{Introduction}

Model checking is a fundamental problem, originally motivated by concerns with the automatic verification of systems, but now more broadly associated with several different fields ranging from Bio-Informatics to Databases to Automated Deduction. In verification settings, model checking problems usually ask whether $S$, a given model of a system, satisfies $\phi$, a given formal property, denoted " $S \models \phi$ ". In [8] we introduced the path model checking problem (see also Open Problem 4.1 in [4]). This problem is unusual since it is a restriction of the classical model checking problem, not an extension as is usually considered. The restriction is that one only considers models having the form of a finite path (or a finite loop, or more generally an ultimately periodic infinite path). These are models without choice, or without nondeterminism. Checking finite paths or loops occurs naturally in many applications: run-time verification [5], analysis of machine-generated scenarios or debugger traces [1], analysis of log files [11], Monte Carlo methods for verification [6], etc.

In [8] we consider path model checking for several temporal logics. Our findings can be summarized as follows:

- checking a deterministic path is usually much easier than checking a nondeterministic structure,

- checking a finite path and checking a loop are usually equivalent (inter-reducible).

Email addresses: markey@lsv.ens-cachan.fr (Nicolas Markey), phs@lsv. ens-cachan.fr (Philippe Schnoebelen). 
In this note, we consider path model checking for the modal $\mu$-calculus. It is known that checking whether a Kripke structure $S$ satisfies a $\mu$-calculus formula (called the branching-time, or $B_{\mu}$, model-checking problem) is PTIME-hard, and is in UP $\cap$ coUP [7]. Additionally, checking whether all paths of $S$ satisfy a $\mu$-calculus formula (called the linear-time, or $L_{\mu}$, model-checking problem) is PSPACE-complete [12].

For path model checking, our findings are surprising:

(1) General $B_{\mu}$ model checking reduces to path model checking. Hence $B_{\mu}$ model checking does not become easier when it is restricted to structures without choice. This does not fit the pattern observed in [8] for other logics like CTL or $\mathrm{CTL}^{*}$.

(2) The above reduction uses loops. We were not able to reduce checking of finite loops to checking of finite paths. Again this does not fit the pattern observed in [8] for other logics.

The paper contains some additional results, e.g., that model checking of finite paths is PTIME-complete (hence the above discrepancies would disappear if it turns out that $\mu$-calculus model checking is in PTIME, a conjecture believed true by several researchers), or relating loops and finite paths in a $\mu$-calculus extended with backwards (sometimes called "past-time") modalities.

\section{Preliminaries}

We refer to [3]. $\mu$-calculus formulae are given by the following grammar:

$$
B_{\mu} \ni \varphi, \psi::=p|\neg p| Z|\varphi \wedge \psi| \varphi \vee \psi|\diamond \varphi| \square \varphi|\mu Z . \varphi| \nu Z . \varphi
$$

where $p$ ranges over a set $\mathrm{AP}$ of atomic propositions, and $Z$ over a set $\mathcal{V}$ of variable names. Our definition only allows negations on propositions, but negation of arbitrary formulae can be defined in the standard way, and similarly for classical shorthands such as $\Rightarrow$, etc. We define the CTL-modalities EF and AG with: $\mathbf{E F} \varphi \stackrel{\text { def }}{=} \mu Z .(\varphi \vee \diamond Z)$ and $\mathbf{A G} \varphi \stackrel{\text { def }}{=} \nu Z .(\varphi \wedge \square Z)$ where $Z$ is any variable not free in $\varphi$.

Formulae in $B_{\mu}$ are interpreted over finite Kripke structures (KS), i.e., labeled finite-state systems of the general form $K=(Q, R, l)$ where $R \subseteq Q \times Q$ is the set of transitions and $l: Q \rightarrow 2^{\mathrm{AP}}$ is the state labeling. As usual, and when $R$ is understood, we write $x \rightarrow y$ rather than $(x, y) \in R$, and we say $y$ is a successor of $x$. Given $S \subseteq Q$, we write $\operatorname{Pre}(S)$ for the set $\{x \in Q \mid \exists y \in S . x \rightarrow y\}$, and $\bar{S}$ for $Q \backslash S$. Then $x \in \overline{\operatorname{Pre}(\bar{S})}$ iff all the successors of $x$ (if any) are in $S$.

Formally, for a $\operatorname{KS} K=(Q, R, l)$ and a context $v: \mathcal{V} \rightarrow 2^{Q}$, the set $\llbracket \varphi \rrbracket_{v}^{K}$ of states 
where $\varphi$ holds is defined inductively:

$$
\begin{aligned}
\llbracket p \rrbracket_{v}^{K} \stackrel{\text { def }}{=}\{x \in Q \mid p \in l(x)\} & \llbracket \neg p \rrbracket_{v}^{K} \stackrel{\text { def }}{=}\{x \in Q \mid p \notin l(x)\} \\
\llbracket \varphi \vee \psi \rrbracket_{v}^{K} & \stackrel{\text { def }}{=} \llbracket \varphi \rrbracket_{v}^{K} \cup \llbracket \psi \rrbracket_{v}^{K} \quad \llbracket \varphi \wedge \psi \rrbracket_{v}^{K} \stackrel{\text { def }}{=} \llbracket \varphi \rrbracket_{v}^{K} \cap \llbracket \psi \rrbracket_{v}^{K} \\
\llbracket Z \rrbracket_{v}^{K} \stackrel{\text { def }}{=} v(Z) & \\
\llbracket \diamond \varphi \rrbracket_{v}^{K} \stackrel{\text { def }}{=} \operatorname{Pre}\left(\llbracket \varphi \rrbracket_{v}^{K}\right) & \llbracket \square \varphi \rrbracket_{v}^{K} \stackrel{\text { def }}{=} \overline{\operatorname{Pre}\left(\overline{\llbracket \varphi \rrbracket_{v}^{K}}\right)} \\
\llbracket \mu Z . \varphi \rrbracket_{v}^{K} & \stackrel{\text { def }}{=} \bigcap\left\{U \subseteq Q \mid \llbracket \varphi \rrbracket_{v[Z \mapsto U]}^{K} \subseteq U\right\} \\
\llbracket \nu Z . \varphi \rrbracket_{v}^{K} & \stackrel{\text { def }}{=} \bigcup\left\{U \subseteq Q \mid U \subseteq \llbracket \varphi \rrbracket_{v[Z \mapsto U]}^{K}\right\}
\end{aligned}
$$

We sometimes omit the " $K$ " and " $v$ " subscripts when no ambiguity arises (or for closed formulae where " $v$ " is irrelevant) and write $x \models_{v}^{K} \varphi$ when $x \in \llbracket \varphi \rrbracket_{v}^{K}$. The above definition entails the following standard fixed-point equalities:

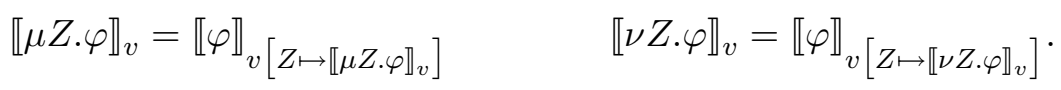

For $\alpha \in \mathbb{N}$, the approximant $\llbracket \mu Z^{\alpha} \cdot \varphi \rrbracket_{v}^{K}$ is defined inductively by

$$
\llbracket \mu Z^{0} \cdot \varphi \rrbracket_{v} \stackrel{\text { def }}{=} \emptyset \quad \text { and } \quad \llbracket \mu Z^{\alpha+1} \cdot \varphi \rrbracket_{v} \stackrel{\text { def }}{=} \llbracket \varphi \rrbracket_{v}\left[Z \mapsto \llbracket \mu Z^{\alpha} \varphi \rrbracket_{v}\right]
$$

Set $\llbracket \nu Z^{\alpha} \cdot \varphi \rrbracket_{v}$ is defined dually. It is well known that, since $K$ is finite, the sequences $\left(\llbracket \mu Z^{\alpha} \cdot \varphi \rrbracket_{v}\right)_{\alpha \in \mathbb{N}}$ and $\left(\llbracket \nu Z^{\alpha} . \varphi \rrbracket_{v}\right)_{\alpha \in \mathbb{N}}$ eventually reach $\llbracket \mu Z . \varphi \rrbracket_{v}$ and $\llbracket \nu Z . \varphi \rrbracket_{v}$ resp.

A KS is deterministic if every state has at most one successor. For such KS's, $\diamond \varphi$ and $\square \varphi$ have very close meanings: $\diamond \varphi$ means that $\varphi$ holds in the successor state, while $\square \varphi$ means that, if there is a successor state, then $\varphi$ holds in that state. We consider below deterministic KS's having the form of a finite path (isomorphic to an initial segment of $\mathbb{N}$, with a last state having no successors), or a finite loop (where there is a single strongly connected component). On loops, the meanings of $\diamond \varphi$ and $\square \varphi$ coincide exactly.

\section{Main result}

Theorem 3.1 $B_{\mu}$ model checking logspace-reduces to model checking of loops.

Hence $\mu$-calculus model checking of loops and general $B_{\mu}$ model checking are equivalent (inter-reducible). Considering deterministic KS's does not simplify the problem:

Corollary 3.2 $B_{\mu}$ model checking of loops is $\mathbf{P T I M E}$-hard, and in $\mathbf{U P} \cap \mathbf{c o U P}$.

The rest of this section describes our reduction. We transform an instance " $x \models^{K}$ $\varphi$ ?" into an equivalent " $x^{\prime} \models^{L} \widetilde{\varphi}$ ?" where $L$ is a loop. We observe that $|L|=O(|K|)$, 


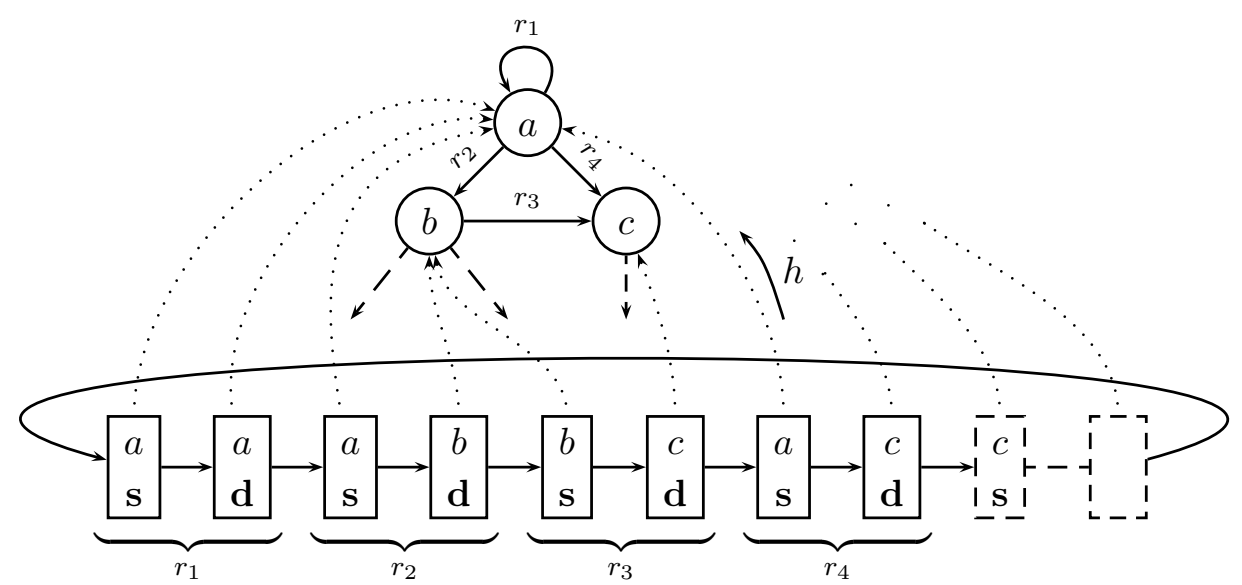

Fig. 1. From non-deterministic to deterministic Kripke structure

and $|\widetilde{\varphi}|=O(|K| \cdot|\varphi|)$. Furthermore, the transformation from $\varphi$ to $\widetilde{\varphi}$ does not increase the alternation depth (Prop. 3.8).

Let $K=(Q, R, l)$ be a KS. For this reduction we assume that $\mathrm{AP}$ and $Q$ coincide, and that $l$ is the identity. ${ }^{1} L$ has labels from $\mathrm{AP}^{\prime} \stackrel{\text { def }}{=} \mathrm{AP} \cup\{\mathbf{s}, \mathbf{d}\}$ where $\mathbf{s}$ (for source) and $\mathbf{d}$ (for destination) are two new atomic propositions. Assume $R=\left\{r_{1}, \ldots, r_{n}\right\}$ contains $n$ transitions: then $L=\left(Q^{\prime}, R^{\prime}, l^{\prime}\right)$ has $Q^{\prime} \stackrel{\text { def }}{=}\left\{s_{1}, d_{1}, s_{2}, d_{2}, \ldots, s_{n}, d_{n}\right\} . R^{\prime}$ has transitions $s_{i} \rightarrow d_{i}$ and $d_{i} \rightarrow s_{(i \bmod n)+1}$ for $1 \leq i \leq n$, arranging $Q^{\prime}$ into a loop. Finally, the labeling $l^{\prime}$ is defined as follows: if $r_{i}=(x, y)$ then $l^{\prime}\left(s_{i}\right)=\{x, \mathbf{s}\}$ and $l^{\prime}\left(d_{i}\right)=\{y, \mathbf{d}\}$.

In summary, $L$ lists the transitions of $K$. The states of $L$ maps to original states via the mapping $h: Q^{\prime} \rightarrow Q$ given by $h\left(x^{\prime}\right)=x \Leftrightarrow x \in l^{\prime}\left(x^{\prime}\right)$. Fig. 1 illustrates this construction on a schematic example.

In the sequel we use $h\left(x^{\prime}\right)$ either as a state or as an element of $\mathrm{AP}^{\prime}$, depending on the context. For any $S \subseteq Q, h\left(x^{\prime}\right) \in S$ iff $x^{\prime} \in h^{-1}(S)$.

Lemma 3.3 Let $S \subseteq Q$. Then $\operatorname{Pre}_{K}(S)=h\left(\llbracket \mathbf{s} \rrbracket^{L} \cap \operatorname{Pre}_{L}\left(h^{-1}(S)\right)\right)$.

PROOF. Assume $x \in \operatorname{Pre}_{K}(S)$ because of a transition $r_{i}$ of the form $x \rightarrow y$ with $y \in S$. In $L, s_{i} \rightarrow d_{i}$ has $d_{i} \in h^{-1}(y) \subseteq h^{-1}(S)$ and $s_{i} \in \llbracket \mathbf{s} \rrbracket^{L}$. Hence $x=h\left(s_{i}\right) \in$ $h\left(\llbracket \mathbf{s} \rrbracket^{L} \cap \operatorname{Pre}_{L}\left(h^{-1}(S)\right)\right)$. Conversely, if $x \in h\left(\llbracket \mathbf{s} \rrbracket^{L} \cap \operatorname{Pre}_{L}\left(h^{-1}(S)\right)\right)$, then $x=h\left(s_{i}\right)$ for some $i$ such that $h\left(d_{i}\right) \in S$. Therefore $r_{i}$ shows that $x \in \operatorname{Pre}_{K}(S)$.

Now, define $\Theta(Z) \stackrel{\text { def }}{=} \bigvee_{x \in Q}[x \wedge \mathbf{E F}(x \wedge Z)]$ and $\Xi(Z) \stackrel{\text { def }}{=} \bigwedge_{x \in Q}[x \Rightarrow \mathbf{A G}(x \Rightarrow Z)]$.

Lemma 3.4 For all $v$, $\llbracket \Theta(Z) \rrbracket_{v}^{L}=h^{-1}\left(h\left(\llbracket Z \rrbracket_{v}^{L}\right)\right)$ and $\llbracket \Xi(Z) \rrbracket_{v}^{L}=\overline{h^{-1}\left(h\left(\overline{\llbracket Z \rrbracket_{v}^{L}}\right)\right)}$.

1 This assumption is no loss of generality. Any general KS can be relabeled in such a way. This requires replacing any proposition used in the original labeling with a disjuction of (the propositions denoting) the states where it holds. This transformation is logspace. 
PROOF. $\llbracket \Theta(Z) \rrbracket_{v}$ is $\bigcup_{x \in Q} \llbracket x \wedge \mathbf{E F}(x \wedge Z) \rrbracket_{v}$. Since $L$ is strongly connected, this is $\left\{x^{\prime} \mid \exists y^{\prime} \in \llbracket Z \rrbracket_{v}, h\left(x^{\prime}\right)=h\left(y^{\prime}\right)\right\}$ by definition of $l^{\prime}$. We end up with $h^{-1}\left(h\left(\llbracket Z \rrbracket_{v}\right)\right)$. The second result follows by duality.

Lemma 3.5 Assume $Y$ and $Z$ are distinct variables. Then for all $v$, we have

$$
\begin{aligned}
& \llbracket \mu Z .(Y \vee \Theta(Z)) \rrbracket_{v}^{L}=\Theta(Y)=h^{-1}\left(h\left(\llbracket Y \rrbracket_{v}^{L}\right)\right) \\
& \llbracket \nu Z .(Y \wedge \Xi(Z)) \rrbracket_{v}^{L}=\Xi(Y)=\overline{h^{-1}\left(h\left(\llbracket Y \rrbracket_{v}^{L}\right)\right) .}
\end{aligned}
$$

PROOF. We only prove the first result, the second one being dual.

$(\subseteq)$ : Write $U$ for $h^{-1}\left(h\left(\llbracket Y \rrbracket_{v}\right)\right)$. Then $\llbracket Y \vee \Theta(Z) \rrbracket_{v[Z \mapsto U]}=\llbracket Y \rrbracket_{v} \cup \llbracket \Theta(Z) \rrbracket_{v[Z \mapsto U]}=$ $\llbracket Y \rrbracket_{v} \cup h^{-1}(h(U))$ (by Lemma 3.4) $=U$. Hence $U$ is a fixed point and $\llbracket \mu Z$. $(Y \vee$ $\Theta(Z)) \rrbracket_{v} \subseteq U$.

$(\supseteq)$ : Write $S$ for $\llbracket \mu Z$. $(Y \vee \Theta(Z)) \rrbracket_{v}$. From the fixed-point property, we have $S=$ $\llbracket Y \vee \Theta(Z) \rrbracket_{v[Z \mapsto S]}=\llbracket Y \rrbracket_{v} \cup \llbracket \Theta(S) \rrbracket_{v}=\llbracket Y \rrbracket_{v} \cup h^{-1}(h(S))$ (by Lemma 3.4). Hence $S \supseteq h^{-1}\left(h\left(\llbracket Y \rrbracket_{v}\right)\right)$.

Thus $\Theta(\psi)$ and $\mu Z .(\psi \vee \Theta(Z))$ are equivalent on $L$ (when $Z$ does not occur free in $\psi)$. The important difference between them is size: $|\Theta(\psi)|$ is in $O(|Q| \cdot|\psi|)$ while $|\mu Z .(\psi \vee \Theta(Z))|$ is in $O(|Q|+|\psi|)$.

We now translate each formula $\varphi$ into a $\tilde{\varphi}$ in such a way that if $\varphi$ holds in $x \in Q$, then $\widetilde{\varphi}$ holds in all $x^{\prime} \in h^{-1}(x)$. Formally, $\widetilde{\varphi}$ is defined inductively by:

$$
\begin{array}{clc}
\widetilde{p} \stackrel{\text { def }}{=} p & \widetilde{\neg p} \stackrel{\text { def }}{=} \neg p & \widetilde{Z} \stackrel{\text { def }}{=} Z \\
\widetilde{\varphi \vee \psi} \stackrel{\text { def }}{=} \widetilde{\varphi} \vee \widetilde{\psi} & \widetilde{\nabla \varphi} \stackrel{\text { def }}{=} \mu Z[(\mathbf{s} \wedge \diamond \widetilde{\varphi}) \vee \Theta(Z)] & \widetilde{\mu Z \cdot \varphi} \stackrel{\text { def }}{=} \mu Z . \widetilde{\varphi} \\
\widetilde{\varphi \wedge \psi} \stackrel{\text { def }}{=} \widetilde{\varphi} \wedge \widetilde{\psi} & \widetilde{\square \varphi} \stackrel{\text { def }}{=} \nu Z .[(\mathbf{s} \Rightarrow \square \widetilde{\varphi}) \wedge \Xi(Z)] & \widetilde{\nu Z . \varphi} \stackrel{\text { def }}{=} \nu Z . \widetilde{\varphi}
\end{array}
$$

Lemma 3.6 For any formula $\varphi$ involving atomic propositions in $A P$, and any context $v: \mathcal{V} \rightarrow 2^{Q}$, and writing $v^{\prime}$ for $h^{-1} \circ v$ :

$$
h^{-1}\left(\llbracket \varphi \rrbracket_{v}^{K}\right)=\llbracket \widetilde{\varphi} \rrbracket_{v^{\prime}}^{L}
$$

In other words, $x^{\prime} \in \llbracket \widetilde{\varphi} \rrbracket_{v^{\prime}}^{L}$ iff $h\left(x^{\prime}\right) \in \llbracket \varphi \rrbracket_{v}^{K}$.

PROOF. By induction on the structure of $\varphi$.

Case $\varphi=p \in \mathrm{AP}$ : Since $\mathrm{AP}=Q$, and by definition of $l^{\prime}, h^{-1}\left(\llbracket p \rrbracket^{K}\right)=\llbracket p \rrbracket^{L}$. 
Case $\varphi=Z \in \mathcal{V}: h^{-1}\left(\llbracket Z \rrbracket_{v}\right)=h^{-1} \circ v(Z)=\llbracket Z \rrbracket_{v^{\prime}}$ by definition of $v^{\prime}$.

Case $\varphi=\mu Z . \psi$ : It is sufficient to show that, for all integers $\alpha, h^{-1}\left(\llbracket \mu Z^{\alpha} . \psi \rrbracket_{v}\right)=$ $\llbracket \mu Z^{\alpha} \cdot \widetilde{\psi} \rrbracket_{v^{\prime}}$. We proceed by induction on $\alpha$. The base case where $\alpha=0$ holds trivially, and the inductive step relies on $h^{-1}\left(\llbracket \mu Z^{\alpha+1} \cdot \psi \rrbracket_{v}\right)=h^{-1}\left(\llbracket \psi \rrbracket_{v\left[Z \mapsto \llbracket \mu Z^{\alpha} \cdot \psi \rrbracket_{v}\right]}\right)=$ $\llbracket \widetilde{\psi} \rrbracket_{h^{-1} \circ v\left[Z \mapsto \llbracket \mu Z^{\alpha} . \psi \rrbracket_{v}\right]}$ by ind. hyp. (Lemma 3.6 on $\left.\psi\right)$. This is $\llbracket \widetilde{\psi} \rrbracket_{v^{\prime}\left[Z \mapsto h^{-1}\left(\llbracket \mu Z^{\alpha} . \psi \rrbracket_{v}\right)\right]}=$ $\llbracket \widetilde{\psi} \rrbracket_{v^{\prime}\left[Z \mapsto \llbracket \mu Z^{\alpha} . \widetilde{\psi} \rrbracket_{v^{\prime}}\right]}$ (by ind. hyp. on $\alpha$ ), hence equals $\llbracket \mu Z^{\alpha+1} . \widetilde{\psi} \rrbracket_{v^{\prime}}$.

Case $\varphi=\diamond \psi: h^{-1}\left(\llbracket \diamond \psi \rrbracket_{v}\right)=h^{-1}\left(\operatorname{Pre}\left(\llbracket \psi \rrbracket_{v}\right)\right)=h^{-1}\left(h\left(\llbracket \mathbf{s} \rrbracket \cap \operatorname{Pre}\left(h^{-1}\left(\llbracket \psi \rrbracket_{v}\right)\right)\right)\right)$ $\left(\right.$ Lemma 3.3) $=h^{-1}\left(h\left(\llbracket \mathbf{s} \rrbracket \cap \operatorname{Pre}\left(\llbracket \widetilde{\psi} \rrbracket_{v^{\prime}}\right)\right)\right)$ by ind. hyp. This is $h^{-1}\left(h\left(\llbracket \mathbf{s} \wedge \diamond \widetilde{\psi} \rrbracket_{v^{\prime}}\right)\right)$, or $\llbracket \widetilde{\nabla \psi} \rrbracket_{v^{\prime}}($ Lemma 3.5).

Remaining cases: The case where $\varphi$ is some $\varphi_{1} \wedge \varphi_{2}$ is obvious and the remaining cases are obtained by duality.

Corollary 3.7 For $x^{\prime} \in h^{-1}(x)$ and $\varphi$ a closed formula, $x \models_{K} \varphi$ iff $x^{\prime} \models_{L} \widetilde{\varphi}$.

PROOF. Lemma 3.6 provides the " $\Rightarrow$ " direction, and the " $\Leftarrow$ " direction too once we observe that $h \circ h^{-1}=I d_{Q}$.

Regarding alternation depth, we refer to [10,2]. A $\mu$-calculus formula is in $\Sigma_{0}\left(=\Pi_{0}\right)$ iff it contains not fixpoint operation. Then, for $n \in \mathbb{N}, \Sigma_{n+1}$ is defined as the smallest class of formulae that contains $\Sigma_{n} \cup \Pi_{n}$ and is closed under conjunctions and disjunctions, $\diamond$ - and $\square$-modalities, least fixed points $\mu Z . \varphi$ with $\varphi \in \Sigma_{n+1}$, and substitution of $\varphi^{\prime} \in \Sigma_{n+1}$ for a free variable of a formula $\varphi \in \Sigma_{n+1}$, provided that no free variable of $\varphi^{\prime}$ is captured by $\varphi$. $\Pi_{n+1}$ is defined dually.

Proposition 3.8 If $\varphi \in \Sigma_{n}$ (or dually, $\left.\Pi_{n}\right)$, then $\widetilde{\varphi}$ is in $\Sigma_{\max (n, 2)}\left(\right.$ resp. $\left.\Pi_{\max (n, 2)}\right)$.

PROOF. By induction on the structure of $\varphi$. The only difficult cases are $\diamond$ and $\square$-formulae. If $\varphi=\diamond \psi$, with $\psi \in \Sigma_{n}$, the induction hypothesis yields that $\widetilde{\psi} \in \Sigma_{\max (n, 1)}$. Then $\widetilde{\varphi}$ is obtained from $\mu Z$. [(s $\left.\left.\wedge \diamond W\right) \vee \Theta(Z)\right]$, a $\Sigma_{1}$-formula, by substituting $\widetilde{\psi}$ for $W$. If $\varphi=\square \psi$, we substitute in a $\Pi_{1}$ (hence $\Sigma_{2}$ ) formula.

\section{Finite paths and acyclic structures}

It is well-known that, for acyclic KS's, $B_{\mu}$ model checking can be done in polynomialtime (hence is PTIME-complete), see, e.g., [9]. Thus model checking finite paths is in polynomial-time and it is not surprising that we could not reduce model checking of loops to model checking of paths: with Theorem 3.1, this would have solved the general $B_{\mu}$ model-checking problem. 
However, even if finite paths seem easier than finite loops, they are not easier than arbitrary acyclic KS's as we now show.

Theorem 4.1 $B_{\mu}$ model checking of finite paths is PTIME-complete.

For this result, it turns out that the reduction from the previous section adapts very easily. If we omit the step $d_{n} \rightarrow s_{1}$ that closed the loop, we obtain a finite path where, assuming that the transitions $R=\left\{r_{1}, \ldots, r_{n}\right\}$ of the acyclic $K$ are given in some topological order, for every vertex of $K$, the destination copies (if any) occur before the source copies. That way, we get:

Lemma 4.2 Given $x^{\prime}, y^{\prime} \in Q^{\prime}$ s.t. $h\left(x^{\prime}\right)=h\left(y^{\prime}\right)$ and $x^{\prime}$ occurs before $y^{\prime}$, for any formula $\varphi \in B_{\mu}$ and any context $v: \mathcal{V} \rightarrow 2^{Q}$, writing $v^{\prime}=h^{-1} \circ v$, we have: if $y^{\prime} \in$ $\llbracket \widetilde{\varphi} \rrbracket_{v^{\prime}}^{K^{\prime}}$, then $x^{\prime} \in \llbracket \widetilde{\varphi} \rrbracket_{v^{\prime}}^{K^{\prime}}$.

That result can easily be shown by induction. We then obtain weaker versions of Lemmas 3.4, 3.5 and 3.6:

Lemma 4.3 Assuming $Y$ and $Z$ are distinct variables, for any context $v^{\prime}$, we have

$$
h\left(\llbracket \Theta(Y) \rrbracket_{v^{\prime}}^{K^{\prime}}\right)=h\left(\llbracket Y \rrbracket_{v^{\prime}}^{K^{\prime}}\right)=h\left(\llbracket \mu Z .\left(Y \vee \Theta(Z) \rrbracket_{v^{\prime}}^{K^{\prime}}\right)\right.
$$

Lemma 4.4 For any formula $\varphi$ of $B_{\mu}$ involving atomic propositions in $A P$, context $v: \mathcal{V} \rightarrow 2^{Q}$, and writing $v^{\prime}$ for $h^{-1} \circ v$ :

$$
\llbracket \varphi \rrbracket_{v}^{K}=h\left(\llbracket \widetilde{\varphi} \rrbracket_{v^{\prime}}^{K^{\prime}} \cap \llbracket \mathbf{s} \rrbracket\right) \quad h^{-1}\left(\llbracket \varphi \rrbracket_{v}^{K}\right) \cap \llbracket \mathbf{d} \rrbracket=\llbracket \widetilde{\varphi} \rrbracket_{v^{\prime}}^{K^{\prime}} \cap \llbracket \mathbf{d} \rrbracket
$$

Now, clearly, a state in $K$ satisfies formula $\varphi$ iff its first source copy in $L$ satisfies $\widetilde{\varphi}$.

\section{Paths, loops, and backwards modalities}

Model checking of loops reduces to finite paths when one considers $2 B_{\mu}$, or " 2 way $B_{\mu}$ ", the extension of $B_{\mu}$ with backwards modalities $\diamond^{-1}$ and $\square^{-1}$. One lets $x \in \llbracket \diamond^{-1} \varphi \rrbracket$ iff there is some $y \in \llbracket \varphi \rrbracket$ with $y \rightarrow x$, and dually for $\square^{-1}$ [13].

Theorem 5.1 The following three problems are logspace inter-reducible:

(a) $B_{\mu}$ model checking of loops,

(b) $2 B_{\mu}$ model checking of loops,

(c) $2 B_{\mu}$ model checking of finite paths.

Corollary 5.2 These three problems are equivalent to $B_{\mu}$ model checking on arbitrary KS's. They are thus PTIME-hard, and in $\mathbf{U P} \cap \mathbf{c o U P}$.

PROOF. (of Theorem 5.1) Since (a) is a special case of (b), we only need two reductions. 
( $\boldsymbol{b}$ reduces to $\boldsymbol{c}$ ) Let $L$ be a loop $x_{1} \rightarrow x_{2} \rightarrow \cdots x_{n}\left(\rightarrow x_{1}\right)$. With $L$, the reduction associates a finite path $F$ of the form $x_{0} \rightarrow x_{1} \rightarrow x_{2} \rightarrow \cdots x_{n} \rightarrow x_{n+1}$. The labeling of $F$ is inherited from $L$ (and irrelevant for $x_{0}$ and $x_{n+1}$ ). The reduction translates a formula $\varphi$ to a $\varphi^{\prime}$ such that $\llbracket \varphi^{\prime} \rrbracket^{F} \backslash\left\{x_{0}, x_{n+1}\right\}=\llbracket \varphi \rrbracket^{L}$. The translation is obtained with

$$
\begin{gathered}
(\diamond \psi)^{\prime} \stackrel{\text { def }}{=} \mu Z \cdot\left(\left(\diamond \psi^{\prime} \wedge \diamond \diamond \top\right) \vee\left(\diamond^{-1}\right)^{n} Z\right) \\
\left(\diamond^{-1} \psi\right)^{\prime} \stackrel{\text { def }}{=} \mu Z \cdot\left(\left(\diamond^{-1} \psi^{\prime} \wedge \diamond^{-1} \diamond^{-1} \top\right) \vee(\diamond)^{n} Z\right)
\end{gathered}
$$

One adds dual clauses for $(\square \psi)^{\prime}$ and $\left(\square^{-1} \psi\right)^{\prime}$, and obvious clauses, like $(\mu Z . \psi)^{\prime} \stackrel{\text { def }}{=}$ $\mu Z .\left(\psi^{\prime}\right)$, for the other constructs. Then $\left|\varphi^{\prime}\right|$ is in $O(|\varphi| \cdot|L|)$.

( $c$ reduces to $\boldsymbol{a}$ ) Let $F$ be a finite path $x_{1} \rightarrow x_{2} \rightarrow \cdots x_{n}$. A loop $L$ is obtained from $F$ by adding a transition $x_{n} \rightarrow x_{1}$ and labeling $x_{1}$ with a new additional proposition i. The reduction then translates a formula $\varphi$ to a $\varphi^{\prime}$ without backwards modalities, and such that $\llbracket \varphi^{\prime} \rrbracket^{L}=\llbracket \varphi \rrbracket^{F}$. We use

$$
(\diamond \psi)^{\prime} \stackrel{\text { def }}{=} \diamond\left(\psi^{\prime} \wedge \neg \mathbf{i}\right) \quad \text { and } \quad\left(\diamond^{-1} \psi\right)^{\prime} \stackrel{\text { def }}{=} \neg \mathbf{i} \wedge \diamond^{n-1} \psi^{\prime}
$$

and obvious remaining clauses. Again, $\left|\varphi^{\prime}\right|$ is in $O(|\varphi| \cdot|L|)$.

\section{Conclusion}

We proved that $\mu$-calculus model checking is not easier when restricting to deterministic Kripke structures having the form of a single loop. On the other hand, we could not reduce model checking of finite loops to model checking of finite paths, a PTIME-complete problem. These results help understand what makes $\mu$-calculus model checking difficult.

It comes as a surprise that none of these two results fits the pattern we exhibited for several other logics [8], where checking nondeterministic KS's is harder than checking deterministic loops, and where finite loops are no harder than finite paths. A possible explanation for the first discrepancy is the expressive power of the $\mu$-calculus, that allows the reduction we developed in Section 3. The second discrepancy is harder to justify, but would disappear if $\mu$-calculus model checking were proved to be in PTIME.

Acknowledgments. We thank Misa Keinänen for drawing our attention to the $\mu$-calculus path model-checking problem.

\section{References}

[1] C. Artho, H. Barringer, A. Goldberg, K. Havelund, S. Khurshid, M. Lowry, C. Pasareanu, G. Roşu, K. Sen, W. Visser, and R. Washington. Combining 
test case generation and runtime verification. Theoretical Computer Science, 336(2-3):209-234, 2005.

[2] J. C. Bradfield. The modal mu-calculus alternation hierarchy is strict. Theoretical Computer Science, 195(2):133-153, 1998.

[3] J. C. Bradfield and C. Stirling. Modal logics and mu-calculi: an introduction. In Handbook of Process Algebra, chapter 4, pages 293-330. Elsevier, 2001.

[4] S. Demri and Ph. Schnoebelen. The complexity of propositional linear temporal logics in simple cases. Information and Computation, 174(1):84-103, 2002.

[5] K. Havelund and G. Roşu. An overview of the runtime verification tool Java PathExplorer. Formal Methods in System Design, 24(2):189-215, 2004.

[6] T. Hérault, R. Lassaigne, F. Magniette, and S. Peyronnet. Approximate probabilistic model checking. In Proc. 5th Int. Conf. Verification, Model Checking, and Abstract Interpretation (VMCAI'04), Venice, Italy, Jan. 2004, volume 2937 of LNCS, pages 73-84. Springer, 2004.

[7] M. Jurdziński. Deciding the winner in parity games is in UP $\cap$ coUP. Information Processing Letters, 68(3):119-124, 1998.

[8] N. Markey and Ph. Schnoebelen. Model checking a path (preliminary report). In Proc. 14th Int. Conf. Concurrency Theory (CONCUR'03), Marseille, France, August 2003, volume 2761 of LNCS, pages 251-265. Springer, 2003.

[9] R. Mateescu. Local model-checking of modal mu-calculus on acyclic labeled transition systems. In Proceedings of the 8th International Conference on Tools and Algorithms for the Construction and Analysis of Systems (TACAS'02), Grenoble, France, April 2002, volume 2280 of LNCS, pages 281-295. Springer, 2002.

[10] D. Niwiński. On fixed point clones. In Proc. 13th Int. Coll. Automata, Languages and Programming (ICALP'86), Rennes, France, July 1986, volume 226 of $L N C S$, pages 464-473. Springer, 1986.

[11] M. Roger and J. Goubault-Larrecq. Log auditing through model checking. In Proc, 14th IEEE Computer Security Foundations Workshop (CSFW'01), pages 220-236, Cape Breton, Nova Scotia, Canada, June 2001. IEEE Comp. Soc. Press.

[12] M. Y. Vardi. A temporal fixpoint calculus. In Proc. 15th ACM Symp. Principles of Programming Languages (POPL'88), San Diego, CA, USA, Jan. 1988, pages 250-259, 1988.

[13] M. Y. Vardi. Reasoning about the past with two-way automata. In Proc. 25th Int. Coll. Automata, Languages, and Programming (ICALP'98), Aalborg, Denmark, July 1998, volume 1443 of LNCS, pages 628-641. Springer, 1998. 